\title{
Total Technology Space Map as a Digital Platform
}

\author{
Jianxi Luo \\ Data-Driven Innovation Lab \\ SUTD-MIT International Design Centre \\ Singapore University of Technology \& Design (SUTD) \\ Email: 1uo@sutd.edu.sg
}

\begin{abstract}
A strand of recent studies utilized complete patent databases and classification systems to construct large network maps of patent technology classes, which might approximate the total technology space. It has been argued that such maps are useful for competitive intelligence analysis, technology road mapping, innovation decision support, and so on in the literature. In this paper, we illustrate the InnoGPS system to integrate such a map with various map-based visual analytic functions for technology navigation, positioning, neighborhood exploration, path finding and information retrieval. These analytics are either descriptive, predictive or prescriptive. During the process of developing InnoGPS, we have conceived a wide spectrum of other potential applications of the total technology space map for consumers, business, education and so on. These possibilities together with the difficulty to construct an accurate technology space representation suggest the strategic value to develop the total technology space map as a digital platform for any applications to discover, manage or represent any data, information and knowledge related to technologies, and to nurture an ecosystem of developers and users.
\end{abstract}

\section{Introduction}

Recent studies in the information science literature have made strides to construct the network maps of patent classes [1]-[4]. In such a network, the nodes represent technology domains and are operationalized as patent classes in a patent classification system, e.g., International Patent Classification (IPC) or Cooperative Patent Classification (CPC), to represent technology domains, e.g., additive manufacturing, biochemistry. The links among different technology domains are weighted according to their knowledge proximity measured based on massive patent co-classification or cross-class referencing records [4][5]. Figure 1 is an example of the patent technology network map, based on all patents (more than 6 million records) from 1974 to 2017 and their citations in the database of the United States Patent \& Trademark Office (USPTO).

In contrast to the patent mining and analysis works focused on the retrieval and analysis of patent samples, such network mapping uses the entire patent database to compute knowledge proximity among all technology classes throughout a patent classification system, in order to provide a complete and accurate picture of the total space of technologies. Hereafter, we call such a network map the total technology space map.

Conceptually, all the technologies that humankind has created to date constitute the total technology space [3]. In the space, two technologies or domains of technologies are proximate if similar knowledge or capabilities are required to design them or are distant if designing them requires distinct knowledge and capabilities. Individuals, firms and regions are often specialized in specific domains in the total technology space. Following learning theories [6], it will be relatively easy for one to understand, learn and adopt the technologies in the proximity of his/her known technologies than those farther away in the technology space. Following the creativity theories [7]-[9], it is relatively easy to synthesize technologies within knowledge proximities to create new technologies, but the less likely synthesis cross large knowledge distances may lead to breakthroughs. Therefore, the total technology space map may provide guidance for learning and creation across technology domains.

It has been argued that such technology space maps have utilities in competitive intelligence analysis, technology road mapping, and innovation decision support [2][4]. Different application examples and cases have been presented in the literature (see Section 2 for a review). One example is the "InnoGPS" system to integrate such a total technology space map with various map-based visual analytic functions for technology space navigation, positioning, neighborhood search, path finding and information retrieval according to knowledge distance (see Section 3 for the example). Meanwhile, the prior works [1]-[4] have presented a variety of patent technology network maps based on 


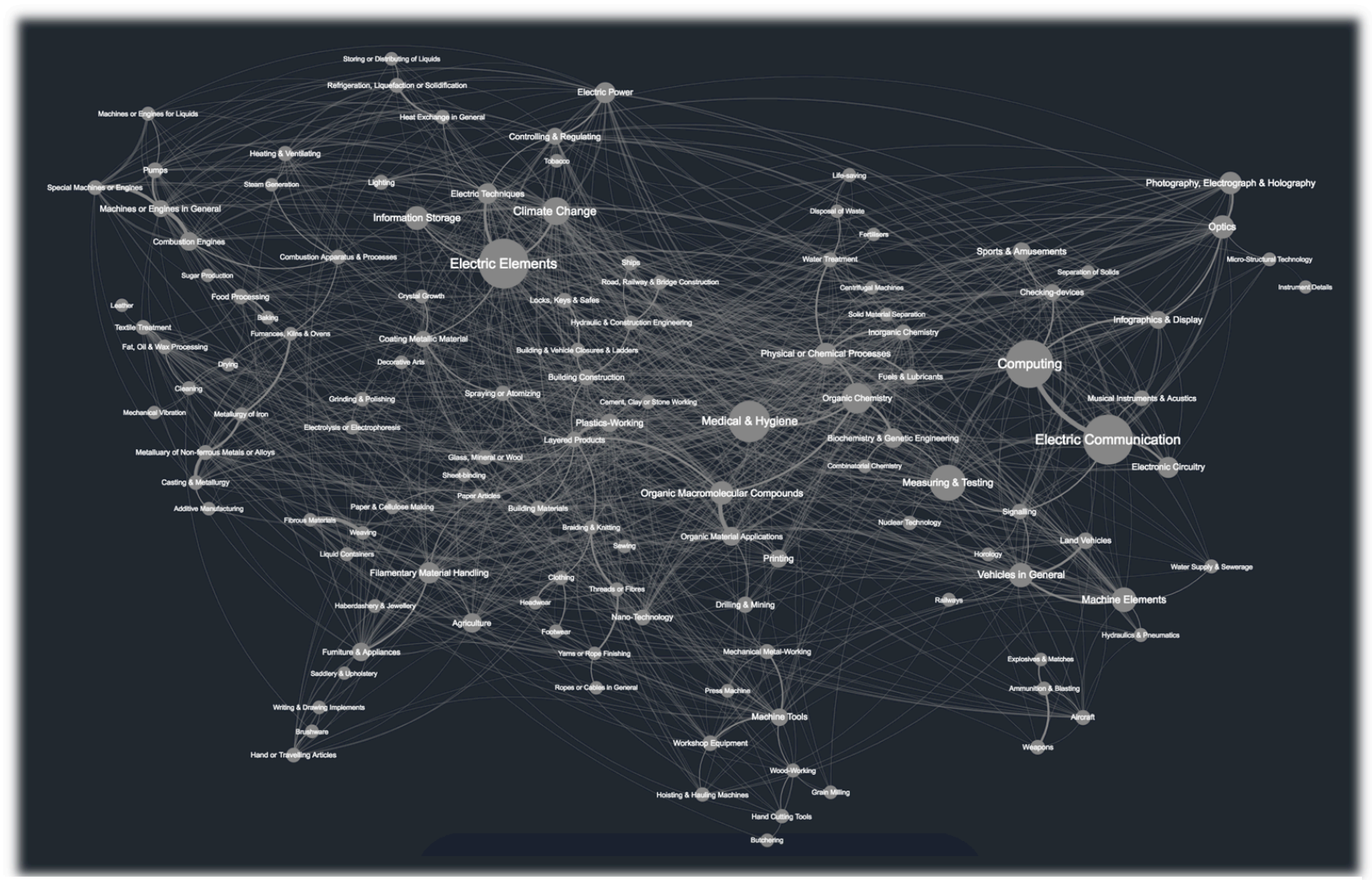

Figure 1. The total technology space map (an example). The nodes are 3-digit international patent classes to represent different technology domains. Network links are weighted according to the knowledge proximity among different domains. Knowledge proximity in this specific case is quantified as the cosine similarity between the vectors of references of the patents in respective domains to other patents in the entire USPTO database. A force-directed algorithm is used to generate the network layout.

different patent classifications (e.g., IPC, CPC), classes at different granularity (e.g., 4- to 7-digits), and different knowledge proximity measures, suggesting the choices but also uncertainty in constructing the total technology space maps.

The diverse potential utilities together with the uncertainty in the creation of the total technology space maps suggest the value to develop a digital platform around the technology space maps to enable an ecosystem of developers and users of a variety of consumer, business and academic applications that make use and make sense of technology-related data, information and knowledge in general. The main objective of this paper is to illuminate the wide application potentials of the total technology space map, and to propose viewing and designing the total technology space map as a digital platform to enable and facilitate the development of the variety of applications.

This paper is organized as follows. In Section 2, we first review different ways to construct the total technology space maps as well as different suggested applications in the literature. In Section 3, we will present the InnoGPS system as an example application of the technology space map. Then we discuss a wide spectrum of possible applications of the total technology space map as a digital platform in Section 4.

\section{Patent technology network maps}

Patent technology network maps with different node and link constructions have been used for the analyses of the movements of different regions [10], firms [11] and individuals [12] in the total technology space.

At the city level, Rigby [13] showed that U.S. cities' entries into and exits from technology domains are highly related to the knowledge proximity among cities' prior and next technology domains. He used USPC (United States Patent Classification) classes to represent technology domains and measured knowledge proximity as the probability that a patent in one class will cite a patent in the other. At the firm level, Breschi et al. [14] found firms in Europe are more likely to diversify across technology domains with high knowledge proximity. They used the patents from European Patent Office and patent co-classification 
codes to measure knowledge proximity between technology domains. Luo et al. [15] used the technology space map to analyze the evolution of technology capability positions of Google with regard to its driverless car project, and found Google followed the strongest network paths to grow its technology positions over time in the total technology space. They used the cosine similarity between the class-to-patent citation vectors to calculate the knowledge proximity.

Alstott et al.'s [12] analysis of 2 million inventors and 4 million patents in the USPTO database showed that inventors are much more likely to explore technology domains that are more proximity to their prior patenting domains. On that basis, they trained a model to predict an inventor's next patenting domains according to his/her prior patent records. They used a normalized knowledge proximity metric by comparing direct empirical patent citations from one domain to another to the same parameter in randomized patent citation networks. Srinivasan et al. [16] used a technology space map to gauge the impact of knowledge distance on design creativity based on a human experiment. They found that engineers are more likely to identify inspirational and useful patents in the domains near their home domains for concept generation, but more novel concepts are inspired by those patents from more distant domains. Their map is based on the Jaccard index of inter-class referencing vectors. Luo et al. [17] further proposed to use a total technology space map as a heuristic ideation tool to enhance design opportunity conception. They used a cosine similarity metric to calculate knowledge proximity and create the map and demonstrate its uses for ideation in a few human experiments.

These prior works have shown the network maps of patent technology classes can be used to evaluate the positions of the patent portfolios of an inventor, firm, region or design domain as a subgraph of the total space map, to assess or predict likelihoods and directions of movements, diversification or evolution of them across different distances in the total space, and to aid in the search for technologies and innovation opportunities. Such analytics are primarily focused on the knowledge proximity between domains. In turn, knowledge proximity enables persons, firms and regions to cross domains for learning, innovation and capability building.

Various knowledge proximity measures have been observed in the literature. Some measures are based on patent reference data, such as the Jaccard index [18] to calculate the count of shared references of a pair of classes normalized by the total count of all unique references of patents in either class [19]. Leydesdorff et al. [1] and Kay et al. [2] used the cosine of the vectors of patent references made from a pair of classes to all other classes respectively. Some other measures are based on the co-classification information, i.e., how often two classes are co-assigned to the same patents [5]. Breschi et al. [14] measured knowledge proximity as the cosine of respective patent classes' vectors of cooccurrences with all other classes in patents. Nesta and Dibiaggio [8] measured the deviation of the actual observed co-occurrences of class pairs in patents from random expectations. Interested readers may refer to a review of the most common knowledge proximity measures used in patent mapping [4].

However, it is unclear which measures are superior and should be chosen for specific purposes and contexts. This ambiguity has limited the validity and actual applications of patent technology network maps. In the following, we describe a more concreate example of the application of the patent technology space map to provide technology and innovation intelligence.

\section{InnoGPS: an application of the technology space map for innovation}

InnoGPS (www.innogps.com) is a cloud-based system developed at the Data-Driven Innovation Lab at Singapore University of Technology \& Design (http://ddi.sutd.edu.sg), and literally is an innovation global positioning system or GPS for innovation. It was introduced in a few recent studies [15][17][20]. Herein, we illustrate it as an exemplar application of the total technology space map. In InnoGPS. The total technology space map is made digitally interactive for the ease of browsing and integrated with various mapbased descriptive, predictive and prescriptive analytic functions, such as positioning technologies (e.g., neural network), individuals (e.g., Robert Langer), firms (e.g., Google) and regions (e.g., Boston, MA), recommending technologies according to knowledge proximity or distance, and technology paths for long-term interests, as well domain-specific technological knowledge discovery. InnoGPS has the following main functions:

Browsing - navigate the map to discover technologies for learning or design inspiration, technical experts for hiring, or companies for collaboration, via viewing the detailed information of companies, inventors and their technologies within each domain (see Figure 2 for such details in the domain "Layered Products"). The information regarding different technologies and domains is organized by knowledge proximity or distance to enhance the heuristic search and the ease of comprehension and learning of new technologies.

Position - locate the technology positions of an innovator, e.g., person, organization, region, or a design practice domain on the technology space map, via mining relevant patent records, and report relevant 
information and statistics within these map positions. In Figure 3 the specific positions of "neural network" are highlighted on the total map, according to the occurrences of the patents related to "neural network" in different classes. The information panel reveals the leading companies (e.g., IBM, the U.S. Navy, Google, Siemens, Hitachi). inventors (i.e., Dharmendra Modha, Eric Hartman, Joseph Bigus, James Keeler, Sunao Takatori), and the most cited and recent patents in the neural network design domain.

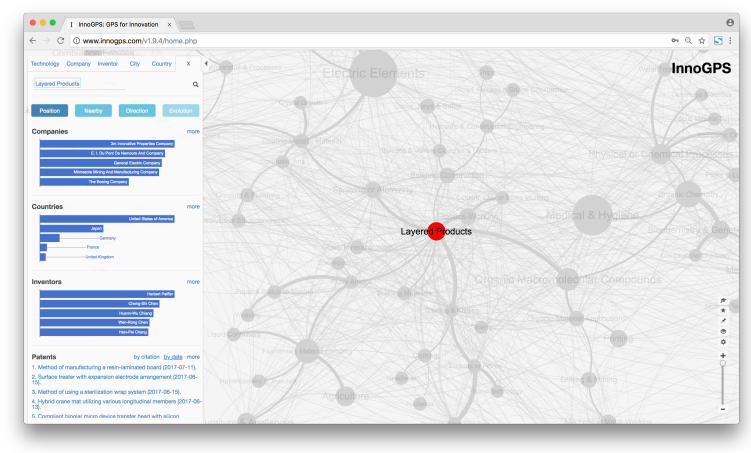

Figure 2. Information retrieved by a single domain

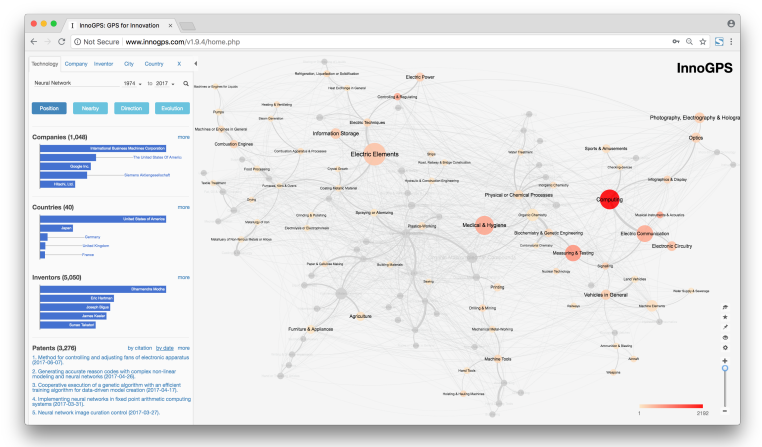

Figure 3. Positions of neural network technologies

Figure 4 uses the red color to highlight the two technology positions of Zoox in 2014, i.e., computing and electric communication. Zoox is an autonomous driving startup based in Menlo Park, CA. It was founded in 2014 by Tim Kentley-Klay and Jesse Levinson and soon valued at US\$1.5 billion in 2016. The information panel in InnoGPS reveals Kentley-Klay and Levinson as the leading inventors from Zoox, as well as the firm's most cited patents to date.

Nearby - identify the most proximate unexplored domains in the white space to the technology positions of an innovator. Due to the high knowledge proximity, such domains can be the most feasible ones for the innovator to enter and are the most obvious sources of inspiration. Figure 5 highlights in purple color the 10 technology domains that Zoox did not start with but are the most proximate to the firm's original technology positions in 2014. Among the 10 domains, "Controlling \& Regulating", "Signaling", "Measuring \& Testing",
"Vehicles in General", "Infographics \& Display", "Checking-devices", and "Music Instruments \& Acoustics" have been already entered by Zoox by the end of 2017 according to its patent records.

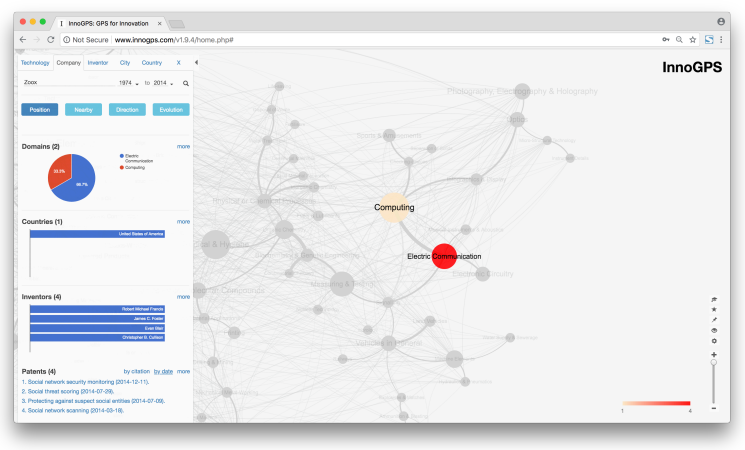

Figure 4. Positions of Zoox in the technology space

Direction - explore the learning and capability building paths from the current technology positions of an innovator to a far domain of long-term interests. Figure 6 shows one technology capability building path (among the alternative ones) for Zoox to consider if they decide to design real vehicles with autonomous driving capabilities. The path suggests that the experts of electric communication within Zoox lead the expansion and that the knowledge and capabilities for "Signaling" are needed before they could effectively design autonomous vehicles.

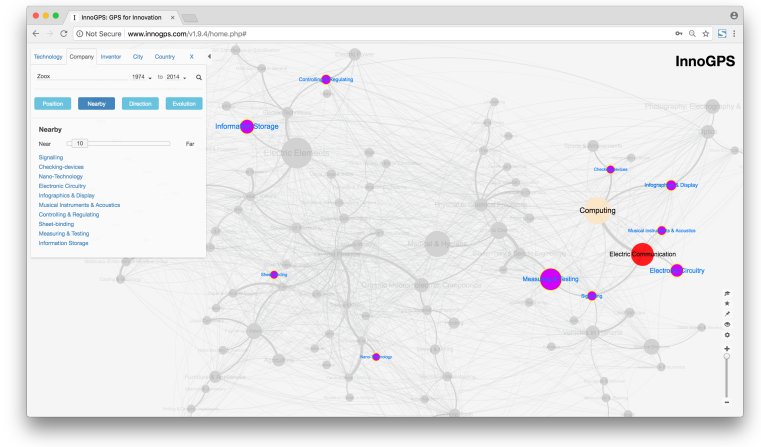

Figure 5. Domains nearby Zoox in 2014

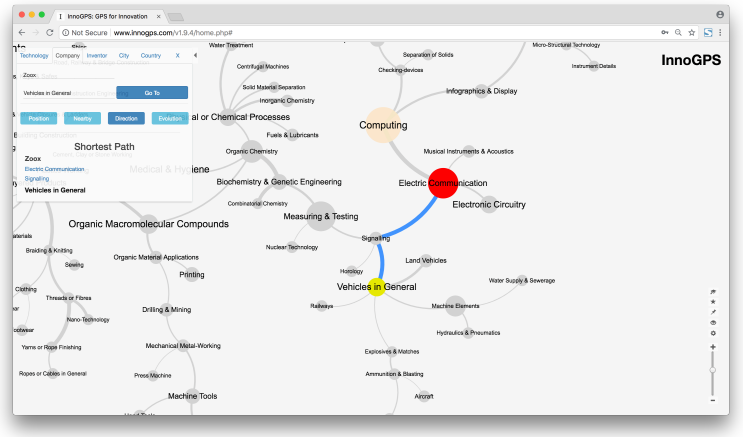

Figure 6. One technology capability building path for Zoox's interest to design autonomous vehicles 
These functions are analogous with those of a traditional GPS system or "Google Maps" that we use for positioning, neighborhood search and direction finding in the physical space. The major difference lies in the maps. InnoGPS is based on a map of the total technology space, whereas Google Maps is based on a geographical map of the physical space. Moreover, InnoGPS goes beyond the prior studies on creating and analyzing patent maps themselves [1]-[4] to provide interactive data-driven visualizations, predictive (e.g., the Nearby function) and prescriptive analytics (e.g., the Direction function). Such interactive visual analytics based on the total technology space map are in line with the recent studies on interactive visual analytics and tools (e.g., ecoxight) for business ecosystem intelligence [21]-[23].

These map-based functions of InnoGPS are specific to the technology space. They are centered on the analysis of knowledge distance and based on creativity theories. New technologies are often created via synthesis, analogy or other fashions of creative transformation of existing technologies [24]-[27]. One's ability to discover, learn, adopt and combine existing but previously-unknown technologies to create new ones is conditioned by the knowledge distance between those unknown but existing technologies and the ones that he/she has already mastered [16][28][29]. One may find it easier and more effective to search and synthesize technologies nearby or within his/her domains of specialization, but more distant domains and technologies may offer more radical innovation opportunities [7]-[9]. As a result, the prescriptive and predictive analytic functions of InnoGPS also address the theoretical understanding of technology searches in the strategy literature [30]-[32].

When one navigates different technology domains on the map for learning, inspiration and cognitive transformation, the theoretically understood influences of knowledge distance on learning and ideation across domains can be programmed into computer algorithms to provide intelligent recommendations of unknown or less known domains and technologies for either attention or caution. For instance, an algorithm can recommend to an innovator a combination of technology domains and the information in them according to the near, moderate and far distances to the innovator's technology positions in the space. Classic machine learning algorithms can be used to detect the innovator's domains of expertise or interests, as well as aptitudes and capacity to cross knowledge distances, based on his/her digital footprints or public records.

Technology recommendations and space navigation guidance may either meet the preferences and aptitudes of an innovator or balance their learning biases. For example, an algorithm may recommend distant technologies to an innovator that has the aptitude for novelty and radicalness but has been only exposed to technologies near his/her familiar domain(s). For a conservative individual, the algorithm may mix distant and proximity technologies in the recommendations to balance his learning and sources of inspiration. Therefore, artificial intelligence capabilities based on learning and creativity theories can be added.

In brief, InnoGPS as a computer-aided tool is aimed to provide artificial intelligence to augment the previously-intuitive human process of technology discovery and learning, as well as the ideation and exploration of innovation opportunities. It presents the potential to change the qualitative and intuitive tradition that innovators rely on to explore new design opportunities, generate innovation ideas and learn new technologies, to a more data-driven, scientifically grounded, and visually-informed fashion.

For engineers, InnoGPS empowers the ideation on next innovation opportunities and career development directions. For startups and SMEs, InnoGPS aids in the search for new product lines, talents and collaborators, for growth and diversification. For large companies and governments, InnoGPS supports technology roadmapping, white space discovery, competitive intelligence, and the search for talents, alliance partners and technologies.

\section{Broad applications of the total technology space map}

InnoGPS was introduced in several prior studies for its uses by technology firms, product engineers, technology entrepreneurs and system designers $[15][17][20]$. The purpose of the foregoing section is to illustrate it as an exemplar application of the total technology space map. However, this application is specific to the interests of a specific type of users who are interested in innovation-related analytics. During the development of InnoGPS, we have conceived a wide spectrum of potential utilities of the total technology space map for more diverse types of consumers, business and academics beyond innovators. These possibilities have further inspired us on developing the technology space map as digital platform to enable the development of a variety of applications by developers from their own sectors. In the following, we first enlist some potential applications, and then discuss why the technology space map can serve as a digital platform shared by these applications.

\section{Applications for individual consumers}


- News and media recommendation - News apps or technology media may manage and recommend technology-related news, articles or other textlabeled contents (Youtube videos, etc.) to readers. Instead of analyzing the direct semantic similarity between documents, one can first position one text document in the total technology space according to its text and the texts of the patent documents in different technology domains and then calculate the distance between the positions of different documents in the total technology space.

- $\quad$ Product recommendation - e-commerce sites (e.g., Amazon, Alibaba) may position individual products (e.g., gadgets, devices, books) in the total technology space, and then calculate and use the knowledge proximity information to retrieve or recommend products to buyers according to their digital footprints. The normal association roles on the ecommerce sites do not consider knowledge distance or proximity between product items in online inventories.

- Web search - search engines (e.g., Google, Bing) may utilize the knowledge proximity information to retrieve and present the technology-related results to the searchers.

\section{Applications for educators and learners}

- Course and faculty management - engineering schools may position their courses and faculty members in the total technology space, then assess the knowledge distance between their positions to guide course portfolio management and faculty recruitment.

- Books and learning materials management university libraries may organize their books and other materials according to their knowledge distance in the total technology space.

- Learning journey management - students may position themselves in the total technology space according to the courses or subjects they have taken and learnt and explore (or get recommendations) about the next subjects to learn given what have been learnt.

\section{Applications for business}

- Data and information management - firms often collect and generate scattered data on technologies and products, and data on employees, clients, suppliers, collaborators, competitors and so on. Such data can be stored and organized in the technology space map and retrieved according to the knowledge distance among the positions of the data items in the technology space.

- Human resources management - a firm may position its technical employees in the total technology space map according to their expertise or project experience and use knowledge distance information to identify engineers suitable for certain projects or build multidisciplinary teams.

- Competitive intelligence - the proximity between the heterogenous positions of different firms may suggest potential competitors, collaborators and acquisition targets.

- Investability analysis - investors may assess the technology positions of an investment target to gauge the value (e.g., growth momentum, expandability), uniqueness and imitability of the firm for growth, differentiation and competition.

- Portfolio management - venture capital or private equity firms may assess the coherence, diversity, expandability of the technology positions of their portfolio companies to guide portfolio adjustment.

- Head hunting - human resource professionals can identify the top technical talents of different distances to the hiring companies in the space.

Generally speaking, one can locate the positions of any text or text-label data item (e.g., webpages, news articles, blogs, reports, books, as well as the descriptions of technologies, products, people, firms and events) in the total technology space, as long as the text can be related to the technical description texts of the patents in each of the classes/domains in the map. Therefore, the total technology space map can serve as a universal infrastructure to organize, make use and sense of the world's growing but scattered data, information and knowledge about technologies according to their knowledge distance, but no more than these. Note that, despite the variety of potential applications, the map will not be useful for problems or applications irrelevant to technologies.

Based on this belief, we propose to build a digital platform around the total technology space map plus APIs (application program interfaces) to enable developers to design innovative applications for better management of data, information and knowledge by distance, in consumption, daily learning, business operations and academics. The core variable that the potential applications needs to call and obtain from the platform is the knowledge proximity or distance among different technologies. However, as suggested in the literature review section earlier, diverse knowledge proximity measures exist in the literature and are far from a convergence.

At the same time, prior studies [4][33] have shown that the link weights and structures of the networks of patent technology classes are rather consistent over time, regardless of the choices of knowledge proximity measures. The stability of such maps might be because the distance between different physical technologies 
(e.g., computing and combustion engine) has an innate physical nature; the technology space is a latent physical existence. It is also found that various proximity measures tend to converge after normalizing the patent technology networks by controlling for impinging factors caused by the human behavior of patenting but not intrinsic properties of the technologies that the patents represent [3]. These results suggest the existence of a unique, innate technology space.

The technology network maps that scholars have created to date are only approximations rather than perfect representations of the unique, innate but latent technology space. By contrast, the geographical maps we use today in Google Maps etc. are considered highly accurate representations of the physical space but have been incrementally improved for hundreds of years. Such mapping improvements were driven by the historical advancements in physical metering technologies and the accumulation of geographical data and knowledge over time. Meanwhile, the maps for the Galaxy and universe are still blur (due to our technical inability by far) to meter such distance and might be as inaccurate as the maps we had for the Earth in the $15^{\text {th }}$ century or earlier. Technology advancements will increase our ability to more accurately measure the distances between farther and farther planets. Likewise, for mapping the technology space, we believe the growing technology-related data and advancing data science and computational technologies may allow us to continually improve the accuracies of the measurement of the innate proximity between different physical technologies in the unique technology space.

Therefore, the main task for the platform builder is to continually search, experiment and improve the knowledge proximity (or distance) measurements and thus increase the accuracy of the technology space maps to approximate the unique, innate technology space that latently conditions human beings' discovery, learning and invention of technologies. Such improvements will in turn enable the development of more useful applications that manage technology-related data, information and knowledge by distance and a related business ecosystem and markets.

\section{Summary}

In this paper, we have reviewed the development of various technology network maps built on patent data and classifications in the information science literature, as well as their utilities and applications. A specific example, i.e., InnoGPS, is given to demonstrate the application value of such a technology space map in aiding innovators in the search for technologies and innovation opportunities and directions. InnoGPS is just one single application example. Meanwhile, we have also conceived a wide range of potential applications of the technology space map for consumers, business and academics. In turn, these ideas suggest a digital platform around the technology space map to enable the development of any applications for the retrieval, management and representation of technology-related data, information and knowledge. The accuracy in measuring knowledge distance and approximating the unique and innate technology space is the key but also challenge for the success of the speculated platform.

\section{References}

[1] Leydesdorff, L., Kushnir, D., and Rafols, I. (2014) Interactive overlay maps for US patent (USPTO) data based on International Patent Classification (IPC). Scientometrics 98(3): 1583-1599.

[2] Kay, L., Newman, N., Youtie, J., Porter, A. L., and Rafols, I. (2014) Patent overlay mapping: Visualizing technological distance. Journal of the Association for Information Science and Technology 65(12): 2432-2443.

[3] Alstott, J., Triulzi, G., Yan, B., and Luo, J. (2017) Mapping technology space by normalizing patent networks. Scientometrics 110(1): 443-479.

[4] Yan, B., and Luo, J. (2017) Measuring technological distance for patent mapping. Journal of the Association for Information Science and Technology 68(2): 423-437.

[5] Engelsman, E. C., and van Raan, A. F. (1994) A patentbased cartography of technology. Research Policy 23(1): $1-26$.

[6] Winston, P. H. (1992) Artificial Intelligence (3rd Edition). Pearson.

[7] Gentner, D., Markman, A. B. (1997) Structure mapping in analogy and similarity. American psychologist 52(1): 45.

[8] Ward, T. B. (1998) Analogical distance and purpose in creative thought: Mental leaps versus mental hops," Advances in analogy research: Integration of theory and data from the cognitive, computational, and neural sciences.

[9] Tseng, I., Moss, J., Cagan, J., and Kotovsky, K. (2008) The role of timing and analogical similarity in the stimulation of idea generation in design. Design Studies 29(3): 203-221.

[10] Boschma, R., Balland, P.-A., and Kogler, D. F. (2014) Relatedness and technological change in cities: the rise and fall of technological knowledge in US metropolitan areas from 1981 to 2010. Industrial and Corporate Change 24(1): 223-250.

[11] Dibiaggio, L., and Nesta, L., (2005), "Patents statistics, knowledge specialisation and the organisation of competencies," Revue d'économie industrielle 110(1): 103-126.

[12] Alstott, J., Triulzi, G., Yan, B., Luo, J (2017) Inventors' explorations across technology domains. Design Science 3: e20.

[13] Rigby, D. L. (2015) Technological relatedness and knowledge space: entry and exit of US cities from patent classes, Regional Studies 49(11): 1922-1937. 
[14] Breschi, S., Malerba, F., Lissoni, F. (2003) Knowledgerelatedness in firm technological diversification. Research Policy 32(1): 69-87.

[15] Luo, J., Yan, B., Wood, K. (2017) InnoGPS for datadriven exploration of design opportunities and directions: The case of Google driverless car project. Journal of Mechanical Design 139(11): 111416.

[16] Srinivasan V., Song, B., Luo, J., Subburaj, K., Rajesh, M. E., Blessing, L., Wood, K. (2018) Does analogical distance affect performance of ideation. Journal of Mechanical Design, In Press.

[17] Luo, J., Song, B., Blessing, L., Wood, K. (2018) Design opportunity conception using technology space map. Artificial Intelligence for Engineering Design, Analysis and Manufacturing. In Press.

[18] Jaccard, P., (1901), "Distribution de la flore alpine dans le bassin des Dranses et dans quelques régions voisines," Bull. Soc. Vaud. Sci. Nat. 37: 241-272.

[19] Von Wartburg, I., Teichert, T., and Rost, K. (2005) Inventive progress measured by multi-stage patent citation analysis. Research Policy 34(10): 1591-1607.

[20] Luo, J. (2018) System-of-systems innovation: Proactive methods for conception and strategies for implementation. Journal of Enterprise Transformation, In Press, DOI: 10.1080/19488289.2017.1409296.

[21] Basole, R. C., Russell, M. G., Huhtamäki, J., Rubens, N., Still, K., \& Park, H. (2015) Understanding business ecosystem dynamics: A data-driven approach. ACM Transactions on Management Information Systems (TMIS), 6(2), 6.

[22] Basole, R. C., Park, H., \& Barnett, B. C. (2015) Coopetition and convergence in the ICT ecosystem. Telecommunications Policy 39(7): 537-552.

[23] Basole, R. C., Srinivasan, A., Park, H., \& Patel, S. (2018) ecoxight: Discovery, Exploration, and Analysis of Business Ecosystems Using Interactive. Visualization.
ACM Transactions on Management Information Systems 9(2): 6.

[24] Weisberg, R. W. (2006) Creativity: Understanding Innovation in Problem Solving, Science, Invention, and the Arts. Wiley.

[25] Arthur, W. B. (2009) The nature of technology: What it is and how it evolves. Simon and Schuster.

[26] Hatchuel, A., and Weil, B. (2009) CK design theory: an advanced formulation. Research in Engineering Design 19(4): 181.

[27] Tang, V., Luo, J. (2013) Idea matrix and creativity operators. Proc. DS 75-7: Proceedings of the 19th International Conference on Engineering Design (ICED13), Design for Harmonies, Vol. 7: Human Behaviour in Design, Seoul, Korea.

[28] Chan, J., Dow, S. P., and Schunn, C. D. (2015) Do the best design ideas (really) come from conceptually distant sources of inspiration? Design Studies 36: 31-58.

[29] Fu, K., Chan, J., Cagan, J., Kotovsky, K., Schunn, C., and Wood, K. (2013) The meaning of "near" and "far": the impact of structuring design databases and the effect of distance of analogy on design output. Journal of Mechanical Design 135(2): 021007.

[30] Stuart, T. E., \& Podolny, J. M. (1996) Local search and the evolution of technological capabilities. Strategic Management Journal 17: 21-38.

[31] Fleming, L. (2001) Recombinant uncertainty in technological search. Management science 47(1): 117132.

[32] Fleming, L., \& Sorenson, O. (2004). Science as a map in technological search. Strategic Management Journal 25(8-9): 909-928.

[33] Hinze, S., Reiß, T., Schmoch, U. (1997) Statistical Analysis on the Distance Between Fields of Technology. Fraunhofer-Inst. Systems and Innovation Research. 